\title{
Many-Body Characterization of Particle-Conserving Topological Superfluids
}

\author{
Gerardo Ortiz, ${ }^{1, *}$ Jorge Dukelsky, ${ }^{2}$ Emilio Cobanera, ${ }^{3}$ Carlos Esebbag, ${ }^{4}$ and Carlo Beenakker ${ }^{3}$ \\ ${ }^{1}$ Department of Physics, Indiana University, Bloomington, Indiana 47405, USA \\ ${ }^{2}$ Instituto de Estructura de la Materia, CSIC, Serrano 123, E-28006 Madrid, Spain \\ ${ }^{3}$ Instituut-Lorentz, Universiteit Leiden, P.O. Box 9506, 2300 RA Leiden, Netherlands \\ ${ }^{4}$ Departamento de Física y Matemáticas, Universidad de Alcala, 28871 Alcala de Henares, Spain
}

(Received 13 July 2014; published 30 December 2014)

\begin{abstract}
What distinguishes trivial superfluids from topological superfluids in interacting many-body systems where the number of particles is conserved? Building on a class of integrable pairing Hamiltonians, we present a number-conserving, interacting variation of the Kitaev model, the Richardson-Gaudin-Kitaev chain, that remains exactly solvable for periodic and antiperiodic boundary conditions. Our model allows us to identify fermion parity switches that distinctively characterize topological superconductivity (fermion superfluidity) in generic interacting many-body systems. Although the Majorana zero modes in this model have only a power-law confinement, we may still define many-body Majorana operators by tuning the flux to a fermion parity switch. We derive a closed-form expression for an interacting topological invariant and show that the transition away from the topological phase is of third order.
\end{abstract}

In recent years, the physics of Majorana zero-energy modes has become a key subfield of condensed matter physics [1-4]. On the theory side, a central result is the bulkboundary correspondence [5] that associates Majorana zero modes to the boundary of (or defects in) a topologically nontrivial superconductor, with the Kitaev chain as a prototypical example [6]. The mathematical formalism underlying this correspondence relies on the symmetries and topological invariants of the Bogoliubov-de Gennes equation [7], a mean-field description of the superconducting state in which the conservation of the number of fermions (a continuous symmetry) is broken down to a discrete symmetry, the conservation of fermion-number parity. Majorana zero modes are directly linked to the spontaneous breaking of this residual discrete symmetry [8].

As the experimental side of Majorana physics continues to develop [9-14], it becomes crucial to unveil how much of the mean-field picture survives beyond its natural limits. This has motivated recent studies [15-21], with the focus on the anomalous $2 \Phi_{0}=h / e$ flux periodicity of the Josephson effect - the hallmark of a topological superconductor [6].

A main thrust of this Letter is the characterization of interacting many-body, number-conserving, topological superconductors, or superfluids, leading to a subsequent analysis on the meaning of Majorana zero modes beyond mean field. The theoretical study of any interacting quantum system is hampered by the exponential growth of the Hilbert space with the number of particles. An additional complication of superconducting systems is the lack of simple principles to guide the design of particlenumber conserving models, in which the phase of the order parameter is not a good quantum number. To overcome both obstacles, we have constructed an exactly solvable, number-conserving variation of the Kitaev chain. Because our model belongs to a class of integrable pairing models [22-24] based on the $s$-wave reduced BCS Hamiltonian first solved by Richardson [25], and on a generalization of the Gaudin magnet [26], we will refer to it as the Richardson-Gaudin-Kitaev (RGK) chain.

The RGK model is integrable for periodic and antiperiodic boundary conditions. It allows us to obtain precise answers for the characterization problem posed here, including the very existence of a topologically nontrivial phase, in an interacting number-conserving system, the order of the phase transition into the trivial phase, the definition of a topological invariant beyond mean-field, and the emergence of many-body Majorana zero modes.

Richardson-Gaudin-Kitaev chain.- Our model is defined by the Hamiltonian

$$
H_{\mathrm{RGK}}=\sum_{k \in S_{k}^{\phi}} \varepsilon_{k} \hat{c}_{k}^{\dagger} \hat{c}_{k}-8 G \sum_{k, k^{\prime} \in \mathcal{S}_{k+}^{\phi}} \eta_{k} \eta_{k^{\prime}} \hat{c}_{k}^{\dagger} \hat{c}_{-k}^{\dagger} \hat{c}_{-k^{\prime}} \hat{c}_{k^{\prime}}
$$

in terms of fermion creation operators $\hat{c}_{k}^{\dagger}$, with momentum $k$-dependent single-particle spectrum $\varepsilon_{k}=-2 t_{1} \cos k-$ $2 t_{2} \cos 2 k$ and interaction strength $G>0$. The interaction is modulated by the potential

$$
\eta_{k}=\sin (k / 2) \sqrt{t_{1}+4 t_{2} \cos ^{2}(k / 2)},
$$

which displays the odd-parity behavior $\eta_{k}=-\eta_{-k}$ characteristic of $p$-wave superconductivity. The pair potential is related to the single-particle spectrum by $4 \eta_{k}^{2}=\varepsilon_{k}+2 t_{+}$, $t_{+}=t_{1}+t_{2}$. This relation is the key to achieve exact solvability, nonetheless, its functional form has been chosen so that it realizes a new model that is physically sound in both momentum and real spaces. 
In real space, we define $c_{j}=L^{-1 / 2} \sum_{k \in \mathcal{S}_{k}^{\phi}} e^{\mathrm{i} j k} \hat{c}_{k}$ for a chain of length $L$, measured in units of the lattice constant. We take $\phi$-dependent boundary conditions $c_{i+L}=e^{\mathrm{i} \phi / 2} c_{i}$. In a ring geometry, periodic boundary conditions $(\phi=0)$ correspond to enclosed flux $\Phi=0$ and antiperiodic boundary conditions $(\phi=2 \pi)$ correspond to $\Phi=\Phi_{0}$. The resulting sets of allowed momenta $\mathcal{S}_{k}^{\phi}$ are $\mathcal{S}_{k}^{0}=\mathcal{S}_{k+}^{0} \oplus \mathcal{S}_{k-}^{0} \oplus\{0,-\pi\}$ and $\mathcal{S}_{k}^{2 \pi}=\mathcal{S}_{k+}^{2 \pi} \oplus \mathcal{S}_{k-}^{2 \pi}$, with $\mathcal{S}_{k \pm}^{0}=L^{-1}\{ \pm 2 \pi, \pm 4 \pi, \ldots$, $\pm(\pi L-2 \pi)\}$ and $\mathcal{S}_{k \pm}^{2 \pi}=L^{-1}\{ \pm \pi, \pm 3 \pi, \ldots, \pm(\pi L-\pi)\}$. The RGK Hamiltonian in real space reads

$$
\begin{gathered}
H_{\mathrm{RGK}}=-\sum_{i=1}^{L} \sum_{r=1}^{2}\left(t_{r} c_{i}^{\dagger} c_{i+r}+\text { H.c. }\right)-2 G I_{\phi}^{\dagger} I_{\phi}, \\
I_{\phi} \equiv 2 \mathrm{i} \sum_{k \in \mathcal{S}_{k+}^{\phi}} \eta_{k} \hat{c}_{k} \hat{c}_{-k}=\sum_{i>j}^{L} \eta(i-j) c_{i} c_{j} .
\end{gathered}
$$

There are at least two cases where the pairing function $\eta(m)$ can be determined in closed form by Fourier transformation of Eq. (2). For $t_{1}=0$ and $t_{2} \neq 0, \eta(m)=$ $\sqrt{t_{2}} \delta_{m 1}$; i.e., we have nearest-neighbor pairing only. For $t_{1} \neq 0$ and $t_{2}=0$ we obtain

$$
\eta(m)=\frac{(-1)^{m} 8 \sqrt{t_{1}}}{\pi} \frac{m}{1-4 m^{2}}, \quad \text { for } L \rightarrow \infty,
$$

so a long-range pairing interaction with a slow $1 / m$ decay with distance $m=i-j$. In general, $\eta(m)$ is a monotonic, decaying function of $m$.

This long-range pairing interaction is the difference with the original Kitaev model [6] that allows for the exact solution beyond the mean-field approximation. As we shall see in a moment, the long-range coupling still allows for a topologically nontrivial phase. It may also be physically relevant for chains of magnetic nanoparticles on a superconducting substrate $[27,28]$, which have recently been shown to support topologically protected Majorana zero modes in the presence of a long-range coupling [29].

Mean-field approximation.-Before we work out the exact solution of the RGK chain, we would like to establish first whether it displays a nontrivial topological phase in the mean-field approximation.

We set $t_{2}=0$ for simplicity and take the pairing interaction Eq. (5). The mean-field approximation to the RGK chain is obtained from the substitution $2 G I^{\dagger} I \rightarrow$ $\Delta^{*} I+\Delta I^{\dagger}$, with gap function $\Delta=2 G\left\langle I_{L}\right\rangle=e^{\mathrm{i} \theta}|\Delta|$. We define Majorana fermion operators $a_{i}=e^{-\mathrm{i} \theta / 2} c_{i}+e^{\mathrm{i} \theta / 2} c_{i}^{\dagger}$, $\mathrm{i} b_{i}=e^{-\mathrm{i} \theta / 2} c_{i}-e^{\mathrm{i} \theta / 2} c_{i}^{\dagger}$. The mean-field Hamiltonian is

$H_{\mathrm{mf}}=\frac{\mathrm{i} t_{1}}{2} \sum_{i=1}^{L-1}\left(b_{i} a_{i+1}-a_{i} b_{i+1}\right)-\frac{\mathrm{i}}{2} \sum_{i>j}^{L} \Delta_{i-j}\left(b_{i} a_{j}+a_{i} b_{j}\right)$,

where $\Delta_{i-j}=|\Delta| \eta(i-j)$, and displays a topological phase characterized by power-law Majorana edge modes and an associated $4 \pi$-periodic Josephson effect. For clarity of presentation, let us compute approximate edge modes to leading order in $\delta_{2}=\Delta_{2} /\left(\Delta_{1}+t_{1}\right)$. The Majorana mode localized at the left end of the chain is $\tilde{a}_{1}=a_{1}+$ $\sum_{i=2}^{L-1} \delta_{i} \sum_{j=2}^{L}\left(-\delta_{2}\right)^{L-j} a_{j}$, so that $\mathrm{i}\left[H_{\mathrm{mf}}, \tilde{a}_{1}\right]=\Delta_{L-1} b_{L}+$ $\mathcal{O}\left(\delta_{2}\right)$. We see that $\Delta_{1}+t_{1}$ controls the localization of the Majorana modes, while $\Delta_{L-1}$ controls the vanishing of the commutator with the Hamiltonian.

Because of the long-range pairing interaction Eq. (5), the wave function of the Majorana modes decays algebraically rather than exponentially in the bulk, and their energy approaches zero as a power law in $1 / L$, similarly to what has been found in other mean-field models based on the Kitaev chain with long-range coupling [29-31].

Exact solution.-To show that the RGK chain is exactly solvable, we rewrite it in the algebraic form

$$
\begin{aligned}
H_{\mathrm{RGK}}= & 8 H_{\phi}+\delta_{\phi, 0}\left(\varepsilon_{0} \hat{c}_{0}^{\dagger} \hat{c}_{0}+\varepsilon_{-\pi} \hat{c}_{-\pi}^{\dagger} \hat{c}_{-\pi}\right) \\
& -4 t_{+} S^{z}+C_{\phi}, \\
H_{\phi}= & \sum_{k \in \mathcal{S}_{k+}^{\phi}} \eta_{k}^{2} S_{k}^{z}-G \sum_{k, k^{\prime} \in \mathcal{S}_{k+}^{\phi}} \eta_{k} \eta_{k^{\prime}} S_{k}^{+} S_{k^{\prime}}^{-},
\end{aligned}
$$

with $C_{\phi}=2 t_{2} \delta_{\phi, 0}, S_{k}^{z}=\frac{1}{2}\left(\hat{c}_{k}^{\dagger} \hat{c}_{k}+\hat{c}_{-k}^{\dagger} \hat{c}_{-k}-1\right)$, and $S_{k}^{+}=$ $\hat{c}_{k}^{\dagger} \hat{c}_{-k}^{\dagger}$ for each pair $(k,-k)$ of pairing-active momenta. These operators satisfy the algebra of SU(2). Hence, $S^{z}=$ $\sum_{k \in \mathcal{S}_{k+}^{\phi}} S_{k}^{z}$ defines a conserved quantity, $\left[H_{\phi}, S^{z}\right]=0$.

The Hamiltonian $H_{\phi}$ belongs to the hyperbolic family of exactly solvable pairing Hamiltonians [24,32], whose best known representative is the chiral $p$-wave superfluid [33-37]. The rational family includes $s$-wave pairing and has been used in the study of the BCS-BEC crossover phenomenon [38]. Eigenstates for $2 M+N_{\nu}$ fermions are

$$
\left|\Phi_{M, \nu}\right\rangle=\prod_{\alpha=1}^{M}\left(\sum_{k \in \mathcal{S}_{k+}^{\phi}} \frac{\eta_{k}}{\eta_{k}^{2}-E_{\alpha}} \hat{c}_{k}^{\dagger} \hat{c}_{-k}^{\dagger}\right)|\nu\rangle .
$$

The state $|\nu\rangle$ with $N_{\nu}$ unpaired fermions satisfies $S_{k}^{-}|\nu\rangle=0$ for all $k$. Moreover, $S_{k}^{z}|\nu\rangle=-s_{k}|\nu\rangle$, with $s_{k}=0$ if the level $k$ is singly occupied or $s_{k}=1 / 2$ if it is empty. The corresponding energy levels are $E_{M, \nu}=\left\langle\nu\left|H_{\phi}\right| \nu\right\rangle+$ $\sum_{\alpha=1}^{M} E_{\alpha}$, with spectral parameters $E_{\alpha}$ determined by the Richardson-Gaudin equations

$$
\sum_{k \in \mathcal{S}_{k+}^{\phi}} \frac{s_{k}}{\eta_{k}^{2}-E_{\alpha}}-\sum_{\beta(\neq \alpha)} \frac{1}{E_{\beta}-E_{\alpha}}=\frac{Q_{\phi}}{E_{\alpha}},
$$

where $Q_{\phi}=1 / 2 G-\sum_{k \in \mathcal{S}_{k+}^{\phi}} s_{k}+M-1$.

In the case of periodic boundary conditions $(\phi=0)$ the two momenta $k=0,-\pi$ are not affected by the interactions and need to be included separately. The eigenvectors then are $\left|\Psi_{N}\right\rangle=\left|n_{0} n_{-\pi}\right\rangle \otimes\left|\Phi_{M, \nu}\right\rangle$, where $n_{0}, n_{-\pi} \in\{0,1\}$ and the total number of fermions is $N=2 M+N_{\nu}+n_{0}+n_{-\pi}$.

The Richardson-Gaudin equations [Eq. (10)] become singular when two or more $E_{\alpha}$ 's approach the same 
single-particle energy $\eta_{k}^{2}$ and also around $E_{\alpha}=0$. At specific values of the interaction strength $G$

$$
G_{n}^{\phi}=\frac{2}{L} g_{n}^{\phi}=\frac{2}{L-2\left(2 M+N_{\nu}\right)+2\left(n+1-\delta_{\phi, 0}\right)},
$$

there are $n$ solutions $E_{\alpha}, 1 \leq n \leq M$, that vanish identically [34,36]. In particular, at $n=M$, where Eq. (9) becomes a pair condensate, $G_{M}^{\phi}$ is precisely the Moore-Read coupling. And, at $n=1$ where $Q_{\phi}=0, G_{1}^{\phi}=G_{c}$ is the Read-Green coupling, associated with the nonanalytic behavior of observables in the thermodynamic limit $[34,36]$.

The numerical solution of Eq. (10) is particularly simple for values of $G>G_{1}^{\phi}$, when all $E_{\alpha}$ 's are real and negative, and for $G=G_{n}^{\phi}$ when $n$ of the $E_{\alpha}$ 's vanish while $M-n$ are real and negative. For other values of $G$ we perform special variable transformations that remove the singularities in Eq. (10), and that is crucial to solve large system sizes. We carried out computations with systems of up to $L \approx 2000$ sites at quarter filling. Without the integrability condition, this would have required diagonalization of a Hamiltonian matrix with the unwieldy dimension $5 \times 10^{242}$. The exact solvability reduces the complexity of the problem to the solution of the $M \simeq 250$ nonlinear coupled equations [Eq. (10)].

We were also able to perform extrapolations to the thermodynamic limit $N, L \rightarrow \infty$ at finite density $\rho=$ $N / L$ and rescaled interaction strength $g=G L / 2$. In that limit, Eq. (10) relate to the mean-field gap and number equations for $H_{\phi}$ [34]

$$
\begin{gathered}
\frac{2 \pi}{g}=\int_{0}^{\pi} \frac{\eta_{k}^{2}}{E_{k}} d k, \quad \rho=\frac{1}{\pi} \int_{0}^{\pi} v_{k}^{2} d k, \\
E_{k}=\sqrt{\left(\frac{\eta_{k}^{2}}{2}-\mu\right)^{2}+\eta_{k}^{2} \Delta^{2},} \quad v_{k}^{2}=\frac{1}{2}-\frac{\eta_{k}^{2}-2 \mu}{4 E_{k}},
\end{gathered}
$$

with quasienergies $E_{k}$ and occupation probabilities $v_{k}^{2}$.

Phase diagram and topological transition.-To establish the quantum phase diagram of the RGK chain, one needs the ground state energy $\mathcal{E}_{0}(\rho, g)$ of $H_{\mathrm{RGK}}$. Depending on the boundary condition and fermion-number parity, one has to consider either $N_{\nu}=0$ or 1 . For periodic boundary conditions, since the levels $k=0,-\pi$ decouple from the rest, $N_{\nu}=0$ for both even and odd $N$. If $N$ is odd, the unpaired particle occupies the $k=0$ level without blocking an active level. For antiperiodic boundary conditions the ground state has $N_{\nu}=0$ for $N$ even, while for $N$ odd it has $N_{\nu}=1$ with blocked level $k_{0}$. The resulting ground state energy is given by

$$
\mathcal{E}_{0}^{\phi}(N)=8 \sum_{\alpha=1}^{M} E_{\alpha}-4 t_{+} M+J_{\phi, 0}+\delta_{N_{\nu}, 1}\left(4 \eta_{k_{0}}^{2}-2 t_{+}\right),
$$

where $J_{\phi, 0}=\delta_{\phi, 0}\left(\varepsilon_{0} \delta_{n_{0}, 1}+\varepsilon_{-\pi} \delta_{n_{-\pi}, 1}\right)$. In the thermodynamic limit the energy density reduces to

$$
e_{0} \equiv \lim _{L \rightarrow \infty} \mathcal{E}_{0}^{\phi} / L=-2 t_{+} \rho-\frac{4}{g} \Delta^{2}+\frac{4}{\pi} \int_{0}^{\pi} \eta_{k}^{2} v_{k}^{2} d k .
$$

The resulting phase diagram is shown in Fig. 1. The RGK chain is gapped for all $g>0$, except for the Read-Green coupling $g_{c}=G_{n=1}^{\phi} L / 2$ where it becomes critical in the thermodynamic limit (without any dependence on the choice of boundary conditions). This critical line defines the phase boundary separating weak (topological) from strong (trivial) pairing phases, and thus is a line of nonanalyticities. At $g_{c}$ a cusp develops in the second derivative of $e_{0}$, that leads to a singular discontinuous behavior of the third-order derivative. Hence, the transition from a weakly paired to a strongly paired superconductor is of third order, just like for the two-dimensional chiral $p$-wave superconductor $[34,36]$.

Open circles in Fig. 1 correspond to the second-order derivative of the exact $e_{0}$ for the antiperiodic RGK chain with $L=2048, N=512$, and $t_{1}=1, t_{2}=0$, obtained by solving Eq. (10) for some selected pairing strength values, and illustrate how close to the thermodynamic limit these system sizes are. We will demonstrate shortly that the weak-pairing phase of the RGK chain is indeed topologically nontrivial.

Fermion parity switches. - We next introduce a quantitative criterion to establish the emergence of topological superconductivity in particle-number conserving, manybody systems. The criterion exploits the behavior of the ground state energy of a system of $N$, and $N \pm 1$ particles, for both periodic and antiperiodic boundary conditions. The emergence of topological order in a superconducting wire, closed in a ring and described in mean field, is associated

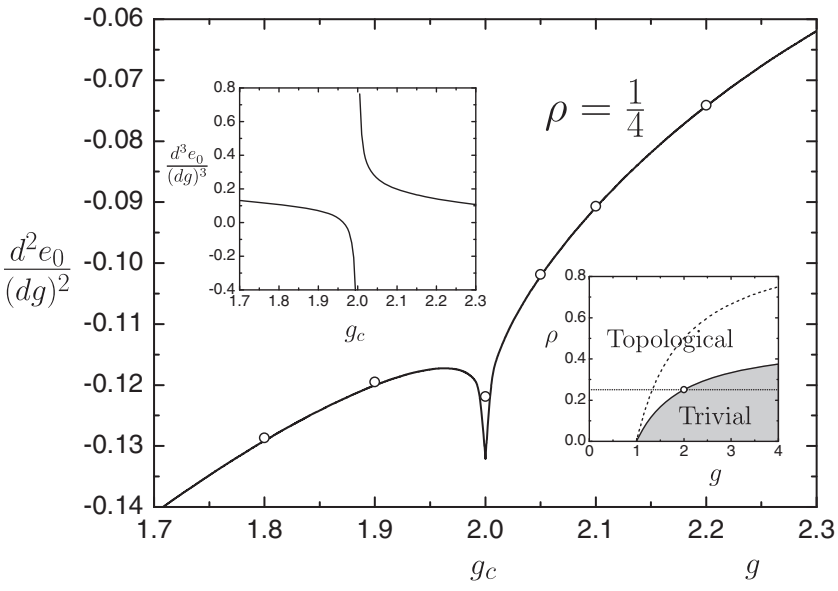

FIG. 1. Third-order phase transition between the topological (weak-pairing) and trivial (strong-pairing) superconducting phases. The continuous line denotes the second order derivative of the ground-state energy density $e_{0}$, evaluated in the thermodynamic limit. Circles are the exact solution for $L=2048$, $N=512$, and antiperiodic boundary conditions. Top inset: Discontinuity in the third-order derivative. Bottom inset: Quantum phase diagram in the $(\rho, g)$-plane. Dashed and full lines represent, respectively, the Moore-Read $\left(g_{M}^{-1}=1-\rho\right)$ and Read-Green $\left(g_{c}^{-1}=1-2 \rho\right)$ boundaries. 
with switches in the ground-state fermion parity $\mathcal{P}(\phi)$ upon increasing the enclosed flux $\Phi=(\phi / 2 \pi) \times \Phi_{0}$ [6,39-44]. Any spin-active superconductor, topologically trivial or not, may experience a crossing of the ground state energies for even and odd number of electrons [45-48]. Regardless of spin, what matters is the number of crossings $N_{X}$ between $\Phi=0=\phi$ and $\Phi=\Phi_{0}, \phi=2 \pi$. The superconductor is topologically nontrivial if $N_{X}$ is odd, otherwise it is trivial.

In the many-body, number conserving, case we need to identify the relevant parity switches signaling the emergence of a topological superconducting phase. Our exact solution gives us access to $\mathcal{P}(\phi)$ only at $\phi=0$ and $\phi=2 \pi$, but this is sufficient to determine whether $N_{X}$ is even or odd. Notice that odd $N_{X}$ means that the flux $\Phi$ should be advanced by $2 \Phi_{0}$-rather than $\Phi_{0}$-in order to return to the initial ground state, which is the essence of the $4 \pi$-periodic Josephson effect $[6,49]$.

To identify the fermion parity switches we calculate the ground state energy $\mathcal{E}_{0}^{\phi}(N)$ for a given number $N$ of fermions in the chain of length $L$, with periodic $(\phi=0)$ or antiperiodic $(\phi=2 \pi)$ boundary conditions, and compare $\mathcal{E}_{0}^{\text {odd }}(\phi)=\frac{1}{2} \mathcal{E}_{0}^{\phi}(N+1)+\frac{1}{2} \mathcal{E}_{0}^{\phi}(N-1) \quad$ and $\quad \mathcal{E}_{0}^{\text {even }}(\phi)=$ $\mathcal{E}_{0}^{\phi}(N)$, where we assumed $N$ even. The difference (inverse compressibility) $\chi(\phi)=\mathcal{E}_{0}^{\text {odd }}(\phi)-\mathcal{E}_{0}^{\text {even }}(\phi)$ determines $\mathcal{P}_{N}(\phi)=\operatorname{sign} \chi(\phi)$, so it has the opposite sign at $\phi=0$ and $\phi=2 \pi$ in the topologically nontrivial phase. We also find that $\mathcal{P}_{N \in \text { even }}(\phi)=-\mathcal{P}_{N \in \text { odd }}(\phi)$ in the topologically nontrivial phase. The results, shown in Fig. 2, unambiguously demonstrate the topologically nontrivial nature of the superconductor for $g<g_{c}$-both in a finite system and

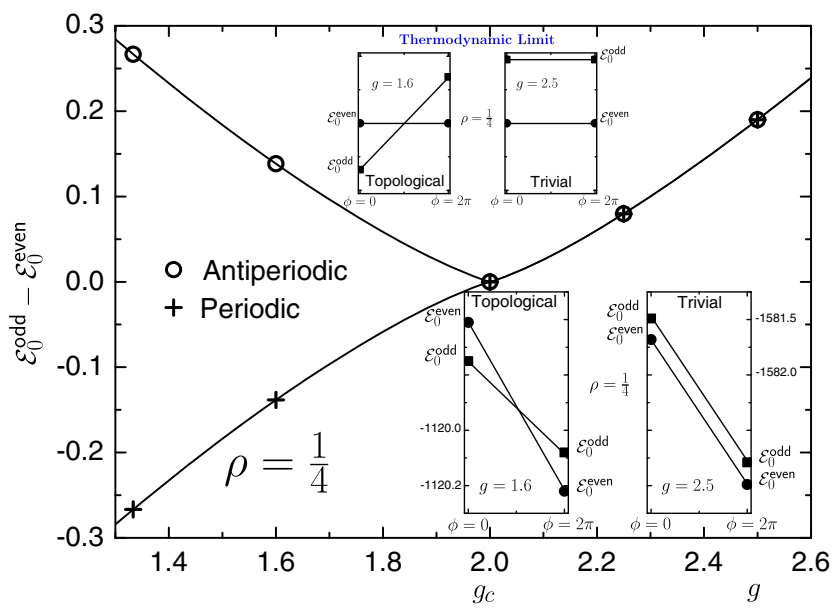

FIG. 2 (color online). Ground state energies for the RGK chain (in units of $t_{1} \equiv 1$, for $\left.t_{2}=0\right)$ for even $(N=2 M)$ and odd $(N=2 M \pm 1)$ number of fermions, and with periodic $(\phi=0)$ or antiperiodic $(\phi=2 \pi)$ boundary conditions. The main plot shows the odd-even difference as a function of the interaction strength $g$ for a finite system (data points, for $N=512, L=2048$ ) and in the thermodynamic limit (continuous lines). The topologically nontrivial state is entered for $g<g_{c}=2$. The insets show the even and odd energies themselves, for the finite system (lower two insets) and in the thermodynamic limit (upper two insets), illustrating the fermion parity switches $\mathcal{P}_{N}(\phi)$. in the thermodynamic limit, and without relying on any mean-field approximation.

The ground state of the odd $(2 M \pm 1)$ system strongly depends on the boundary conditions. For periodic boundary conditions the unpaired particle always occupies the $k_{0}=0$ level, while for the antiperiodic case it starts blocking the Fermi momentum $k_{F}=k_{0}$ at $g=0$, continuously decreasing its modulus with increasing $g$, up to $k_{0}=\pi / L$ at $g_{0} \sim$ $1.1936(\rho=1 / 4)$, corresponding to $\mu=\Delta^{2}$ in the thermodynamic limit. In that limit $\chi(\phi)$ has a particularly simple form: $\chi(0)=-8 \mu$, and $\chi(2 \pi)=8|\mu|$ for $g>g_{0}$.

Topological invariant and zero modes. - In addition to the parity switches, one would like to have an independent way of signaling a topological phase transition. The occupation number $\mathcal{N}_{k=0}$ of the $k=0$ single-particle state is a topological invariant [34,50], being the onedimensional analogue of the winding number in two space dimensions [51,52]. By combining the integrals of motion [24,34] with the Hellmann-Feynman theorem we find

$$
\begin{gathered}
\mathcal{N}_{k}=\frac{1}{2}-s_{k}-4 s_{k} \gamma^{2} \sum_{\alpha=1}^{M} \frac{\eta_{k}^{2}}{\left(\eta_{k}^{2}-E_{\alpha}\right)^{2}} \frac{\partial E_{\alpha}}{\partial \gamma}, \\
\gamma=\frac{G_{c} W(G)}{3 W(G)-2 G_{c}}, \quad W(G)=\frac{G_{c} G}{2 G-G_{c}} .
\end{gathered}
$$

The mapping $W$ satisfies $W(W(G))=G$, with fixed point $W\left(G_{c}\right)=G_{c}$, typical of self-dual transformations. In the thermodynamic limit, $\lim _{L \rightarrow \infty} \mathcal{N}_{k=0}=\Theta\left[G_{c}-G\right]$ with $\Theta[x]$ the unit step function, thus signaling the topological transition at $G=G_{c}$, as can be confirmed numerically.

It is known for the mean-field case that it is possible to construct exact zero-energy modes by tuning the flux to a fermion parity switch [53]. We may generalize this concept to our many-body system, and thus give meaning to the notion of many-body Majorana modes. By varying the boundary conditions in the topologically nontrivial phase the "ground state" energies $\mathcal{E}_{0}^{\text {odd }}(\phi)$ and $\mathcal{E}_{0}^{\text {even }}(\phi)$ cross at some critical $\phi^{*}$. (They must cross, because their order is inverted at $\phi=0$ and $\phi=2 \pi$.) At this value of $\phi=\phi^{*}$ one can identify Majorana zero modes as follows. Define the normalized ground states $\left|\Psi_{0}^{\text {even }}\right\rangle=\left|\Psi_{0}^{N}\right\rangle, \quad\left|\Psi_{0}^{\text {odd }}\right\rangle=$ $\left(\left|\Psi_{0}^{N+1}\right\rangle+\left|\Psi_{0}^{N-1}\right\rangle\right) / \sqrt{2}$, with the transition operator $\hat{T}=\left|\Psi_{0}^{\text {even }}\right\rangle\left\langle\Psi_{0}^{\text {odd }}\right|$. Clearly, $\hat{T}^{2}=0$, and $\left\{\hat{T}, \hat{T}^{\dagger}\right\}=\hat{P}_{0}$, with $\hat{P}_{0}=\left|\Psi_{0}^{\text {even }}\right\rangle\left\langle\Psi_{0}^{\text {even }}|+| \Psi_{0}^{\text {odd }}\right\rangle\left\langle\Psi_{0}^{\text {odd }}\right|$ the projector onto the ground-state subspace. Then, the corresponding Majorana operators are: $\Gamma_{1}=\hat{T}+\hat{T}^{\dagger}, \quad \mathrm{i} \Gamma_{2}=\hat{T}-\hat{T}^{\dagger}$, $\left\{\Gamma_{1}, \Gamma_{2}\right\}=0$, modes that have an operational meaning for open quantum systems. In the isolated chain with open boundaries, we would expect the equivalent of these modes to be localized at the edges due to the bulk energy gap. It would be interesting to check this numerically.

In conclusion, we have constructed a variation of the Kitaev Hamiltonian that is both number conserving and interacting, but still exactly solvable. This allowed us to identify the fermion parity switches needed for 
characterizing topological superconductivity in generic interacting many-body systems, integrable or not, and to analyze the meaning of many-body Majorana modes. In turn, this implies that the fractional, i.e., the lack of a $\Phi=\Phi_{0^{-}}$ periodic, Josephson effect can indeed serve as an experimental probe in these systems. We have shown that our Richardson-Gaudin-Kitaev model shares the features of the mean-field Kitaev model that have made it a paradigm of topological superconductivity. There is one difference, the long-range nature of the pairing interaction, but in view of recent experimental developments $[28,29]$, this may be a welcome feature of the model rather than a drawback.

J. D. and C. E. are supported by Grant No. FIS201234479 of the Spanish Ministry of Economy and Competitiveness. E. C. and C. B. acknowledge support by the Netherlands Organization for Scientific Research (OCW/NWO/FOM) and an ERC Synergy Grant.

*ortizg@indiana.edu

[1] M. Z. Hasan and C. L. Kane, Rev. Mod. Phys. 82, 3045 (2010).

[2] X.-L. Qi and S.-C. Zhang, Rev. Mod. Phys. 83, 1057 (2011).

[3] J. Alicea, Rep. Prog. Phys. 75, 076501 (2012).

[4] C. W. J. Beenakker, Annu. Rev. Condens. Matter Phys. 4, 113 (2013).

[5] S. Ryu, A. P. Schnyder, A. Furusaki, and A. W. W. Ludwig, New J. Phys. 12, 065010 (2010).

[6] A. Yu. Kitaev, Phys. Usp. (Suppl.) 44, 131 (2001).

[7] P.-G. de Gennes, Superconductivity of Metals and Alloys (Benjamin, New York, 1966).

[8] B. van Heck, E. Cobanera, J. Ulrich, and F. Hassler, Phys. Rev. B 89, 165416 (2014).

[9] V. Mourik, K. Zuo, S. M. Frolov, S. R. Plissard, E. P. A. M. Bakkers, and L. P. Kouwenhoven, Science 336, 1003 (2012).

[10] A. Das, Y. Ronen, Y. Most, Y. Oreg, M. Heiblum, and H. Shtrikman, Nat. Phys. 8, 887 (2012).

[11] M. T. Deng, C. L. Yu, G. Y. Huang, M. Larsson, P. Caroff, and H. Q. Xu, Nano Lett. 12, 6414 (2012).

[12] A. D. K. Finck, D. J. Van Harlingen, P. K. Mohseni, K. Jung, and X. Li, Phys. Rev. Lett. 110, 126406 (2013).

[13] H. O. H. Churchill, V. Fatemi, K. Grove-Rasmussen, M. T. Deng, P. Caroff, H. Q. Xu, and C. M. Marcus, Phys. Rev. B 87, 241401 (2013).

[14] T. D. Stanescu and S. Tewari, J. Phys. Condens. Matter 25, 233201 (2013).

[15] A. M. Tsvelik, arXiv:1106.2996.

[16] M. Cheng and H.-H. Tu, Phys. Rev. B 84, 094503 (2011).

[17] F. Hassler and D. Schuricht, New J. Phys. 14, 125018 (2012).

[18] A. Manolescu, D. C. Marinescu, and T. D. Stanescu, J. Phys. Condens. Matter 26, 172203 (2014).

[19] J. D. Sau, B. I. Halperin, K. Flensberg, and S. Das Sarma, Phys. Rev. B 84, 144509 (2011).

[20] L. Fidkowski, R. M. Lutchyn, C. Nayak, and M. P. A. Fisher, Phys. Rev. B 84, 195436 (2011).

[21] B. van Heck, F. Hassler, A. R. Akhmerov, and C. W. J. Beenakker, Phys. Rev. B 84, 180502(R) (2011).

[22] L. Amico, A. Di Lorenzo, and A. Osterloh, Phys. Rev. Lett. 86, 5759 (2001).
[23] J. Dukelsky, C. Esebbag, and P. Schuck, Phys. Rev. Lett. 87, 066403 (2001).

[24] G. Ortiz, R. Somma, J. Dukelsky, and S. M. A. Rombouts, Nucl. Phys. B707, 421 (2005).

[25] R. W. Richardson, Phys. Lett. 3, 277 (1963).

[26] M. Gaudin, J. Phys. (Paris) 37, 1087 (1976).

[27] T.-P. Choy, J. M. Edge, A. R. Akhmerov, and C. W. J. Beenakker, Phys. Rev. B 84, 195442 (2011).

[28] S. Nadj-Perge, I. K. Drozdov, B. A. Bernevig, and A. Yazdani, Phys. Rev. B 88, 020407 (2013).

[29] F. Pientka, L. I. Glazman, and F. von Oppen, Phys. Rev. B 88, 155420 (2013).

[30] D. Vodola, L. Lepori, E. Ercolessi, A. V. Gorshkov, and G. Pupillo, Phys. Rev. Lett. 113, 156402 (2014).

[31] W. DeGottardi, M. Thakurathi, S. Vishveshwara, and D. Sen, Phys. Rev. B 88, 165111 (2013).

[32] J. Dukelsky, S. Pittel, and G. Sierra, Rev. Mod. Phys. 76, 643 (2004).

[33] M. I. Ibañez, J. Links, G. Sierra, and S. Y. Zhao, Phys. Rev. B 79, 180501(R) (2009).

[34] S. M. A. Rombouts, J. Dukelsky, and G. Ortiz, Phys. Rev. B 82, 224510 (2010).

[35] C. Dunning, M. Ibanez, J. Links, G. Sierra, and S.-Y. Zhao, J. Stat. Mech. (2010) P08025.

[36] S. Lerma H., S. M. A. Rombouts, J. Dukelsky, and G. Ortiz, Phys. Rev. B 84, 100503(R) (2011).

[37] The real space properties of these two-dimensional models have not been studied. Although they share the same algebraic realization of the hyperbolic family, both kinetic and potential (pairing) energy terms are different from the ones in the RGK chain.

[38] G. Ortiz and J. Dukelsky, Phys. Rev. A 72, 043611 (2005).

[39] A. Keselman, L. Fu, A. Stern, and E. Berg, Phys. Rev. Lett. 111, 116402 (2013).

[40] C. W. J. Beenakker, J. M. Edge, J. P. Dahlhaus, D. I. Pikulin, S. Mi, and M. Wimmer, Phys. Rev. Lett. 111, 037001 (2013).

[41] J. D. Sau and E. Demler, Phys. Rev. B 88, 205402 (2013).

[42] A. Haim, A. Keselman, E. Berg, and Y. Oreg, Phys. Rev. B 89, 220504 (2014).

[43] F. Crépin and B. Trauzettel, Phys. Rev. Lett. 112, 077002 (2014).

[44] C. W. J. Beenakker, arXiv:1407.2131.

[45] A. Sakurai, Prog. Theor. Phys. 44, 1472 (1970).

[46] A. V. Balatsky, I. Vekhter, and J.-X. Zhu, Rev. Mod. Phys. 78, 373 (2006).

[47] W. Chang, V. E. Manucharyan, T. S. Jespersen, J. Nygård, and C. M. Marcus, Phys. Rev. Lett. 110, 217005 (2013).

[48] E. J. H. Lee, X. Jiang, M. Houzet, R. Aguado, C. M. Lieber, and S. De Franceschi, Nat. Nanotechnol. 9, 79 (2014).

[49] K. Sengupta, I. Zǔtić, H.-J. Kwon, V. M. Yakovenko, and S. Das Sarma, Phys. Rev. B 63, 144531 (2001).

[50] S. S. Botelho and C. A. R. Sà de Melo, J. Low Temp. Phys. 140, 409 (2005).

[51] G. E. Volovik, Sov. Phys. JETP 67, 1804 (1988).

[52] M. S. Foster, M. Dzero, V. Gurarie, and E. A. Yuzbashyan, Phys. Rev. B 88, 104511 (2013).

[53] A. R. Akhmerov, J. P. Dahlhaus, F. Hassler, M. Wimmer, and C. W. J. Beenakker, Phys. Rev. Lett. 106, 057001 (2011), [Supplemental material.] 